\title{
DIE LARVALE ENTWICKLUNG VON CHIROCENTRUS DORAB (Forsk.)
}

VON

\author{
Dr. H. C. DELSMAN.
}

(Mit 6 Figuren im Tekst)

Wer sich mit dem Studium der Lebensweise indischer Fische befassen will, hat dabei den grossen Vorteil, sich beim Bestimmen der Species auf die zahlreichen Arbeiten BLEEKER's stützen zu können, während ihm das Determinieren ganz besonders erleichtert wird durch das von dichotomischen Tabellen versehene „Fishes of the Indo-Australian Archipelago" von WEBER und DE BEAUFORT, wovon sich nur hoffen lässt, dass die Autoren, wie umfassend das Unternehmen auch sei, es einmal ganz vollendet sehen mögen, den indischen Ichthyologen zu Nutz und Frommen. Weniger sind wir bekannt mit der Biologie und mit der Fortpflanzung und Entwicklung der indischen Fische, besonders der Seefische. Es war mir, der ich mich immer besonders für die Entwicklungsgeschichte so der Wirbellosen wie auch der Wirbeltiere interessiert habe, daher eine wirkliche Freude, als mir am Bataviaschen Laboratorium für Meeresforschung die Gelegenheit geboten wurde, die Eier und Larven der Fische der Javasee zu studieren.

In Europa hat man mit diesem Studium schon bedeutende Fortschritte gemacht, seitdem im Jahre 1864, SARS die ersten pelagischen Fischeier, nämlich diejenigen des Dorsches, entdeckte. Besonders für die Fische der Nord- und Ostsee verfügen wir über ziemlich vollständige Angaben, wie aus der Zusammenfassung EHRENBAum's in „Nordisches Plankton” (1905 und 1909) deutlich zutage tritt. Es hat sich gezeigt, dass die meisten Nordseefische pelagische Eier haben, während die Zahl derjenigen, deren Laich auf den Boden sinkt oder an Steinen und Algen befestigt wird, sehr beschränkt ist.

$\mathrm{Ob}$ es nun in Indien je gelingen wird, die verschiedenen Arten pelagischer Eier und Fischlarven so vollständig kennen zu lernen, muss sehr bezweifelt werden. Erstens ist die Zahl der Arten ungleich viel grösser als in europäischen Gewässern. Dazu kommt, dass in Europa jede Art ihre bestimmte Laichzeit hat, während das in den Tropen, so weit ich aus meinen bisherigen Fängen habe schliessen können, nicht der Fall ist. Mehrere leicht erkennbare Eiern, auch wenn ich sie noch nicht näher habe bestimmen können, fand ich das ganze Jahr hindurch, ohne dass von einer Periodizität etwas zu verspüren war. Während also in Europa das Vorkommen verschiedener Eier zeitlich getrennt ist, findet man in indischen Gewässern alle möglichen Arten das ganze Jahr hindurch, was die Determinierung nicht leichter macht.

Manche Eigentümlichkeiten europäischer Formen findet man bei den pelagischen Eiern in der Javasee, worauf sich meine Untersuchungen beschränken, wieder. Durch eine längliche Form zeichnen sich die Eier der verschiedenen Sardellen-ähnlichen Fischen aus. Ebenso wie das Ei des europäischen Leyerfisches (Callionymus lyra) eine netzförmige Zeichnung feiner Leisten auf der Eimembran aufweist, so ist das auch mit einigen Eiern aus der Javasee der Fall, welche ich übrigens noch nicht näher bestimmt habe. An ihrem zerklüfteten Dotter lassen sich die Eier der Clupeiden und Aal-artigen Fische leicht erkennen, welche letzteren sich weiter durch ihre Grösse und durch den ansehnlichen Durch- 
messer der geräumigen Eihülle auszeichnen. Ein letztes Merkmal ist die An- oder Abwesenheit von Oeltröpfen im Dotter. Hierin unterscheiden sich oft nahe verwandte Arten. So traf ich Aal-Eier ohne Oeltröpfen, solche mit einem grossen Oeltropfen und solche mit vielen kleinen Oeltröpfen an.

Der einfachste Weg, die verschiedenen Eier kennen zu lernen, wäre nun zweifellos die künstliche Befruchtung. Damit habe ich indessen leider gar keinen Erfolg gehabt. Es ist sehr schwierig, wirklich laichreife Fische zu bekommen, was vielleicht damit zusammenhängt, dass das Laichen nicht an eine bestimmte Periode gebunden ist. Meine Versuche in diese Richtung habe ich daher vorläufig aufgegeben.

Jetzt wird mit einem grossen vertikalen Eiernetze horizontal längs der Oberfläche gefischt, während der Untersuchungsdampfer „Brak” möglichst langsam fährt. So wird jedesmal etwa eine Stunde gefischt. Aus dem Fang werden sofort mit einem Leseglase und einer Pipette die Eier ausgesucht. Jede Art wird in ein eigenes Glas mit Meerwasser gebracht, wo die Eier zum Ausschlüpfen kommen und die Larven möglichst weit aufgezogen werden. Wenn der Dotter aufgezehrt ist und die Augen schwarz geworden sind, bleiben die Larven gewöhnlich nicht lange mehr am Leben. Leider ist die Determinierung dann in weitaus den meisten Fällen noch nicht möglich, so dass wir versuchen müssen, Anschluss an pelagisch gefischte Larvenstadien zu gewinnen.

Ein grosser Vorteil beim Studium der pelagischen Eier in dieser Weise ist nun der Umstand, dass man in den Fängen gewöhnlich eine grössere Zahl einer bestimmten Art antrift, oft auch dieselbe Art in mehreren auf einander folgenden Fängen. Dazu kommt, dass gewöhnlich alle Eier sich in demselben Entwicklungsstadium befinden. Dies macht es möglich, aus einem einzigen Fange eine vollständige Entwicklungs-Serie herzustellen, indem man z. B. jeden Morgen und jeden Abend ein oder einige Exemplare in Seewasser-Formaldehyd bringt. Die Entwicklung schreitet in den Tropen viel schneller fort als in höheren Breiten. Während in der Nordsee die mittlere Temperatur des Wassers $10^{\circ}$ beträgt, ist sie für die Javasee auf nicht weniger als $28^{\circ} \mathrm{zu}$ stellen. Dieser Unterschied hat natürlich einen beschleunigenden Einfluss auf den Entwicklungsprozess. Indem z. B. ein Sardellenei in der Südersee 3 Tage zum Ausschlüpfen braucht, geschieht das bei den javanischen Arten wahrscheinlich innerhalb 24 Stunden. Während der Nacht abgelegt, schlüpfen die Jungen schon am Abend des ersten Tages aus, wie ich es bei mehreren Arten beobachtet habe.

Beim Studium der pelagischen Eier liegt es nun vor der Hand, uns zuerst an die grösseren und am leichtesten kennbaren Arten zu wenden. Dazu gehört zweifellos das Ei von Chirocentrus dorab. Dieser Fisch hat das Aeussere eines sehr in die Länge gezogenen Herings mit zugeschärfter Bauchseite, was ihm den malayischen Namen: „parang-parang” oder "golok-golok” (= Kappmesser) besorgt hat. Seine Länge beträgt gewöhnlich $60-70$ c.M. In der Javasee kommt er allgemein vor und auf dem Fischmarkt zu Batavia ist er eine gewöhnliche Erscheinung.

Die Eier lassen sich an verschiedenen Eigentümlichkeiten leicht erkennen. Erstens gehören sie zu den grösseren Arten, da der Diameter des lebenden Eies $1590-1670 \mu$ beträgt. Zweitens ist die Eihaut nicht glatt, sondern zeigt auf ihrer Oberfläche eine netzförmige Zeichnung von bienenwabenartig angeordneten Leisten. Der Parang-parang stimmt hierin also überein mit dem Leyerfisch (Callionymus lyra) aus nordischen Meeren und mit mehreren anderen Fischen aus der Javasee, welche oben erwähnt wurden, und wobei es auch eine (übrigens noch unbekannte) Art giebt, deren Eier ungefähr den gleichen Durchmesser haben als diejenigen des Chirocentrus. Die letzteren unterscheiden sich von allen diesen jedoch dadurch, dass die Maschen hier ungleich viel feiner sind. Dieselben haben einen Durchmesser von noch nicht $15 \mu$ und lassen sich daher in einer Abbildung wie Fig. 1 nicht gut wiedergeben.

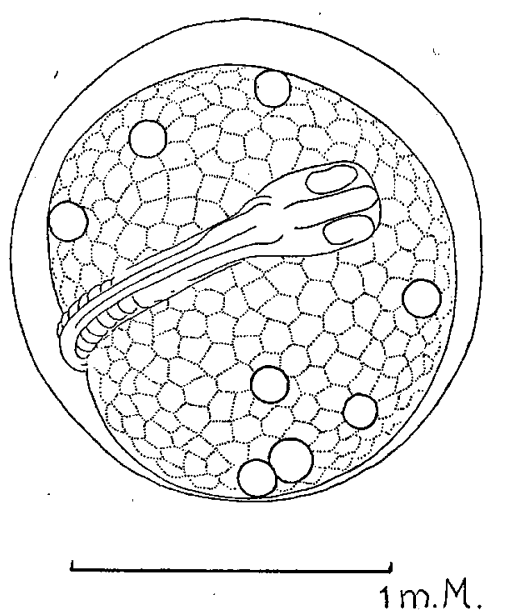

Fig, 1. Ei von Chirocentrus dorab (die wabenähnliche Zeichnung der Eihaut ist zu fein um angegeben zu werden).

Eine dritte Eigentümlichkeit ist der segmentierte Dotter, wie er so kennzeichnend ist für die Eier der hering- und aalartigen Fische und der immer einen deutlichen Hinweis dafür giebt, in welche Richtung wir nach der Herkunft eines pelagischen Eies zu suchen haben. 
Unregelmässig im Dotter verbreitet finden sich eine Anzahl kleiner, farbloser Oeltröpfen, wovon ich in verschiedenen Eiern von 7 bis 19 zählte. Die Zahl ist also nicht constant.

Nach dem Vorhergehenden erweist sich also das Ei des Chirocentrus dorab als eines der am leichtesten kennbaren pelagischen Eier der Javasee.

Wie lange die Entwicklung innerhalb des Eies nimmt, habe' ich leider noch nicht ermitteln können. Mehrere Male habe ich eine grössere Zahl dieser Eier aus den horizontalen Oberflächenfängen isoliert, aber alle waren sie schon so weit entwickelt, dass die Anlage des Embryo sichtbar war. Nach meinen Erfahrungen bei anderen, verwandten Eiern schätze ich die Entwicklungsdauer auf $1 \frac{1}{2}-2$ Tage.

Die Zeit des Ausschlüpfens war immer dieselbe, nämlich um 8 bis 9 Uhr Abends, und wir dürfen hieraus schliessen, dass auch die Laichzeit an einer gewissen Tageszeit gebunden ist, obgleich wir nicht näher wissen, welche diese ist.

Eine Betrachtung der eben ausgeschlüpften Larve bestätigt unsere Vermutung, dass̀ wir es hier mit dem Ei eines den heringartigen verwandten Fisches zu tun hatten. Der sehr weit nach hinten gelegene After zusammen mit der allgemeinen Gestalt und dem segmentierten Dotter lassen keinen Raum für Zweifel. Der post-anale Körperabschnitt macht, wie aus Fig. 2 hervorgeht weniger als 1/r

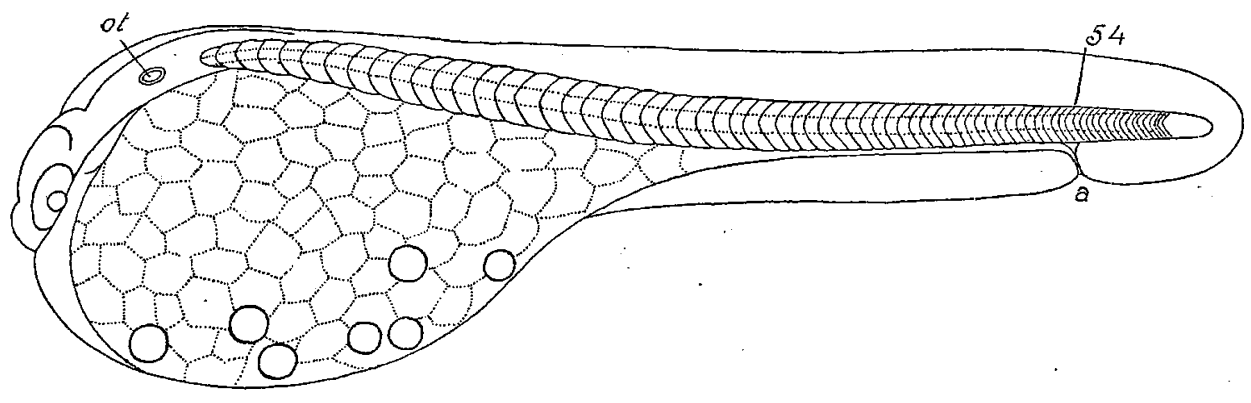

Fig. 2. Eben ausgeschlüpfte Larve. Verg, wie Fig. 1. $a$ anus.

der ganzen Körperlänge aus, obgleich bei etwas älteren Larven dieses Verhältnis sich allmählich zugunsten des Schwanzes ändert, sodass es in Fig. 3 schon 1 auf $5 \frac{1}{2}$ beträgt. Der Kopf hebt sich noch nicht von dem umfangreichen Dottersack $a b$, eine Mundöffnung, ein Unterkiefer und Kiemenspalten fehlen noch. Die Augen sind noch farblos. Die Anlage der statischen Organe ist in der Gestalt zweier Bläschen, worin je zwei stark lichtbrechende Statolithen, anwesend. Das Herz hatte schon im Ei zu pochen angefangen.

In den ersten Stunden nach dem Ausschlüpfen erfolgt eine bedeutende Streckung des Embryo, so dass die Länge der in Fig. 3 abgebildeten Larve, welche übrigens schon 48 Stunden alt ist, bald annähernd erreicht wird. Der Dotter nimmt allmählich ab, die Oeltröpfen werden kleiner. Der Kopf

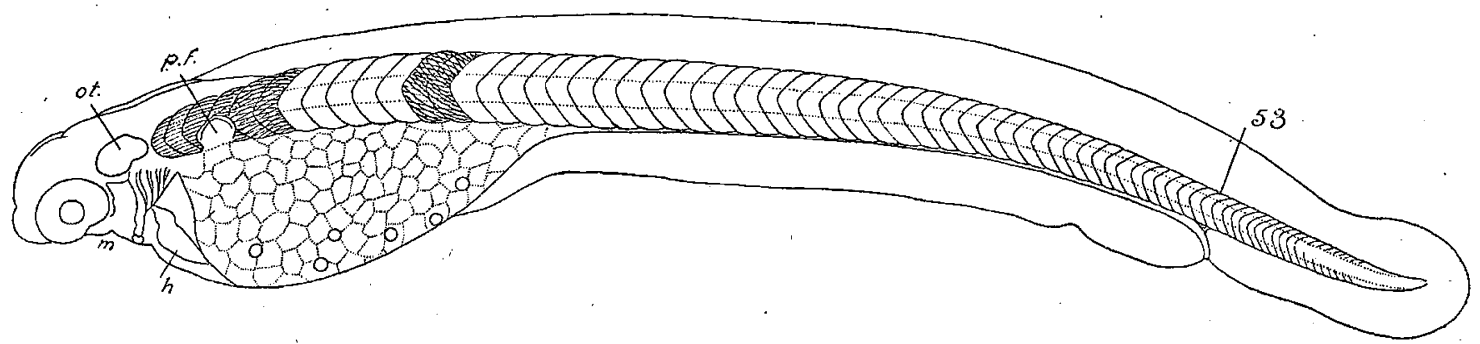

Fig. 3. Larve von 48 Stunden. Vergr. wie vorige Fig. $m$ Mund. $h$ Herz, ot Ohrbläschen, $p f$. Brustflosse.

hebt sich mehr ab, die Anlagen der Kiemenspalten und des Mundes werden sichtbar. Die Anlage der Brustflosse tritt auf zwischen dem 3. und 4. Myotom. (Fig. 3, pf).

Bezeichnend für alle Larven von Clupea-artigen Fischen, welche ich bis jetzt aus pelagisch gefischten Eiern aus der Javasee gezogen habe, ist der gekreuzte Verlauf der Muskelfasern innerhalb der Myotomen, wie ich in Fig. 3 anzugeben versucht habe. In eben ausgeschlüpften Larven lässt sich diese Eigentümlichkeit schon constatieren. Die Muskelfasern laufen nicht, wie z. B. bei den Larven aalartiger Fische, parallel der Längsachse des Tieres, sondern in zwei sich kreuzenden Richtungen. Eine nähere Untersuchung mit höherer und tieferer Einstellung des Mikroskops bei stärkerer Vergrösserung lehrt, 
dass die Muskelfasern in drei Schichten angeordnet sind, wovon die oberflächliche auf die untere Hälfte des Myotoms beschränkt ist, die tiefste dagegen auf die obere Hälfte, während die mittlere Schicht sich auf das ganze Myotom von oben bis unten erstreckt. In der mittlern Schicht laufen nun die Muskelfasern von vorn unten nach hinten oben; in den beiden anderen Schichten umgekehrt. Nur in den vordersten Myotomen, hart hinter dem Ohrbläschen, lässt sich dieser gekreuzte Verlauf der Fasern nicht beobachten.

In allen diesen Hinsichten stimmt also die Larve überein mit denjenigen von Clupea-Arten und verwandten Formen, wie ich deren viele aus dem Ei gezüchtet habe. Für die weitere Bestimmung wollen wir auf die Zahl der Myotomen acht geben. Die Gesamtzahl der Myotomen bietet wenig Halt. Das vorderste Myotom, dicht hinter dem Ohrbläschen, lässt sich immer mit Leichtigkeit bestimmen, das hinterste jedoch nicht, weil im Schwanze die Myotomen allmählich kleiner und weniger deutlich werden, um schliesslich in die undifferenzierte Schwanzknospe überzugehen. Obgleich sich oft auch noch mit ziemlicher Gewissheit das letzte Myotom bestimmen lässt, so haben wir doch keine Gewissheif darüber, ob nicht während der weiteren Entwicklung der jungen Larve noch neue gebildet werden.

Wir können also unsere Aufmerksamkeit mehr auf die Rumpf- oder prä-analen Myotome richten. Wie verhält sich deren Zahl zu derjenigen der Rumpfwirbel im erwachsenen Fische? Hierüber habe ich nur sehr wenig in der mir zur Verfügung stehenden Literatur finden können. Es ist sehr zu bedauern, dass in den zahlreichen Abbildungen in Ehrenbaum's „Nordisches Plankton, Eier und Larven von Fischen" die Zahl der Myotomen nirgends mit Genauigkeit angegeben ist und dieselbe auch fast nirgends erwähnt wird. Es ist möglich, dass in der Literatur zerstreut noch einige Angaben zu finden wären. Ich habe dieselbe jedoch hier nur sehr unvollständig zu meiner Verfügung. Im Allgemeinen entsteht ein Wirbel auf der Grenze von je zwei Myotomen, aber wie es dabei mit den vordersten Myotomen, auf der Grenze von Schädel und Wirbelsäule geht, ist, so weit mir bekannt, für die Teleostier noch nicht festgestellt.

Eine zweite Frage ist, ob die Lage des Anus als constant betrachtet werden darf oder ob eine gewisse Verschiebung desselben nach vorn oder nach hinten möglich ist. Beim Züchten meiner Larven habe ich oft den Eindruck bekommen, dass die Lage sehr constant war. Bei Fistularia ${ }^{1}$ ). zum Beispiel, wo die Zahl der Myotomen doch gross ist, fand ich in auffolgenden Stadien den Anus immer unter dem 50. Myotom.

Dennoch lässt sich hierauf nicht trauen, wie aus SchmidT's ²) Beobachtungen an Aallarven hervorgeht. Besonders bei den Conger-Arten fand er während der Entwicklung eine starke Zunahme der Zahl der prä-analen Myotomen. Bei Conger vulgaris z. B. betrug die Zahl derselben bei einer Länge von 9 m.M. 89, bei einer Länge von 12,5 m.M. schon 100, um dann weiter zu steigen bis 125 , bei einer Länge von 74 m.M. Zu gleicher Zeit nimmt die Zahl der Schwanzmyotomen beträchtlich ab, sodass wir es offenbar mit einer Verschiebung des Anus nach hinten zu tun haben. Es muss hier gleich bemerkt werden, dass bei anderen Arten, z. B. bei Muraena helena, die Lage des Afters sich wieder viel konstanter erwies. Bei vier Larven dieser Art, lang resp. 9, 12, $17 \frac{1}{2}$ und $44 \frac{1}{2}$ m.M., erwies sich die Zahl der prä-analen Myotomen immer genau 80, welche Zahl schon im Ei erreicht wird. Bei einem erwachsenen Exemplar wurden freilich nur 70 prä-anale Wirbel gezählt.

Wie es nun hierum bei den heringartigetl Fischen steht, darüber habe ich leider keine Angaben finden können. Auf meiner Bitte war jedoch Dr. REDEKE, von Helder, so liebenswürdig mir eine Anzahl Larven von Südersee-Heringen zu schicken. Wie Dr. REDEKE mir schrieb, giebt HEINCKE in seiner "Naturgeschichtẹ des Herings" (1898), welche Arbeit ich hier nicht zur Verfügung habe, für die mittlere Zahl der Schwanzwirbel dieser Heringrasse 13,9 (s. Tab. 115). Wo die Zahl der Wirbel 55,5 beträgt, finden wir für die mittlere Zahl der Rumpfwirbel ungefähr $55,5 \div 14=41,5$. Nun zählte ich in 3 eben ausgeschlüpften Larven, wovon eine noch mit einem Dottersack, und alle ungefähr 8 oder 9 m.M. messend, 47 prä-anale und \pm 15 post-anale Myotomen, und in einer älteren Larve, fast 20 m.M. lang, 45 Rumpf- und \pm 15 caudale Myotomen. Aus diesen Beobachtungen lässt sich folgern, dass auch hier die Lage des Afters nicht konstant ist, sondern dass eine vorwärtsche Wanderung stattfindet.

1) Delsman, Fish Eggs and Larvae from the Java Sea, in: Treubia, Vol. II, 1921.

2) Meddelelser fră Kommissionen for Havundersögelser, Serie: Fiskeri, Bd. 4, nr. 2, S. 4. 
Bei eben ausgeschlüpften Larven von Chirocentrus dorab fand ich nun 53 bis 54 prä-anale Myotomen, und die selben Zahlen bei Larven von 48 Stunden, wie in Fig. 3 abgebildet. Viel weiter ist es mir nicht gelungen, die Larven zu züchten, aber anschliessende Stadien fand ich in den Fängen. Es zeigte sich, dass die Zahl der Rumpfmyotomen anfänglich ein wenig grösser wīrd. Bei zwei noch ein wenig älteren Larven mit noch farblosen Augen und noch nicht völlig verbrauchtem Dotter fand ich 55, bei Larven von ungefähr derselben Länge (6,8 -7 m.M.), aber mit schwarzen Augen (Fig. 4)

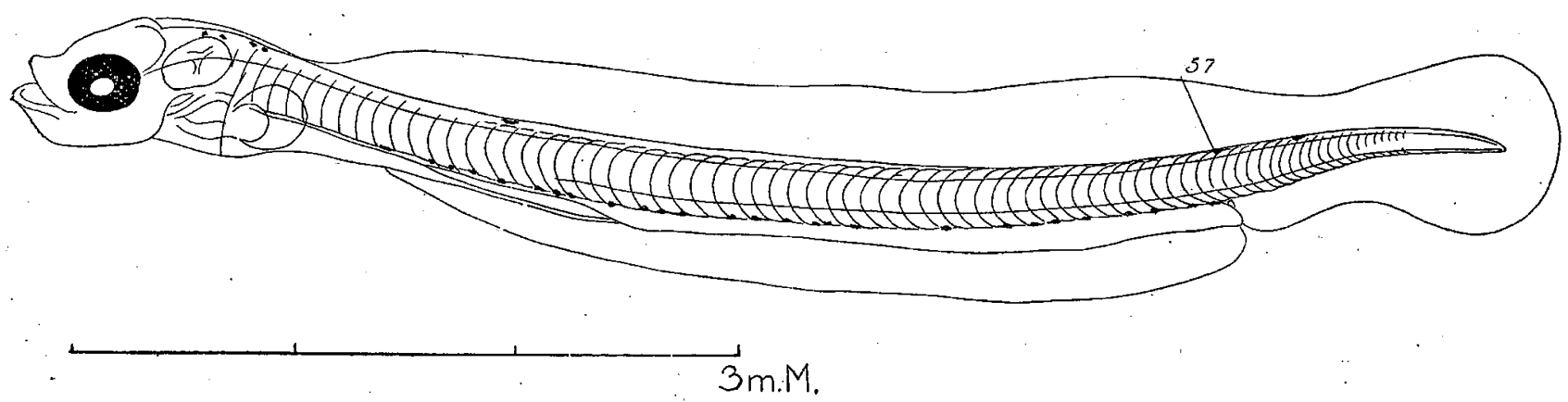

Fig. 4. Pelagisch gefischte Larve von $7 \mathrm{~m} . M$.

57-58 Myotomen vor dem Anus. Darauf fängt die Zahl allmählich zu sinken an. Bei 3 Larven von $12^{3} / 4$ m.M. fand ich noch 57, bei einer von $13 \frac{1}{2}$ und einer von 18 m.M. je $56-57$ Myotomen (es lässt sich nicht immer genau bestimmen, welches Myotom wir als das letzte Rumpfmyotom zu betrachten haben), bei einer von 20,5 m.M. (Fig. 6) 54 und bei einer von 22 m.M. 52 Myotomen. Aeltere Larven habe ich noch nicht beobachtet. Konstant ist die Zahl der prä-analen Myotomen und folglich die Lage des Afters auch hier also nicht. Hinter dem Anus konnte ich in jüngeren Stadien gewöhnlich noch etwa 16 bis 17 Myotomen zählen, bei einem Stadium, wie der Fig. 6, bis 19, wobei die undifferenzierte Zellmasse am Ende also nicht einbegriffen ist.

Vergleiche ich die hier beschriebene Larve mit anderen von mir gezüchteten Larven von heringartigen Fischen aus der Javasee, so lässt sich jedenfalls constatieren, dass die Zahl der Myotomen eine

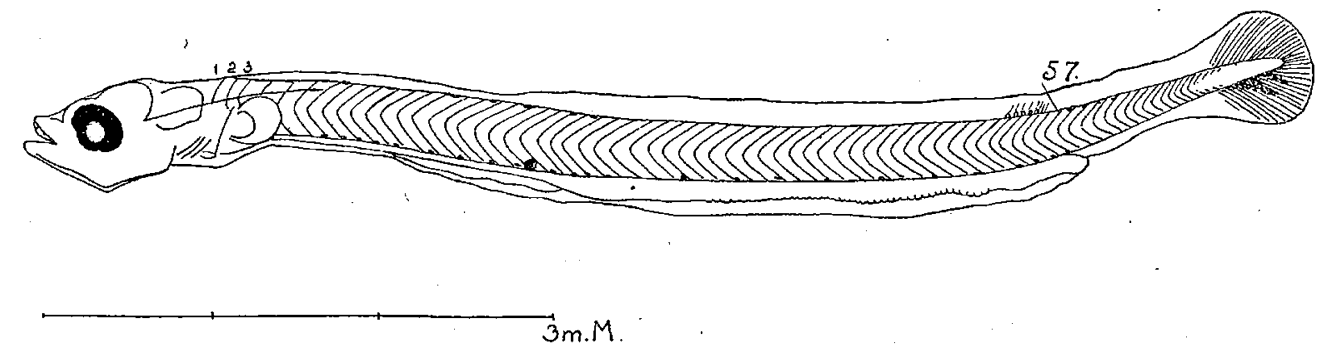

Fig. 5. Pelagisch gefischte Larve von 7 m.M. mit Anlage von Schwanz- und Rückenflosse.

sehr grosse ist. Bei vier Larvenarten, die ich aus Eiern vom Typus desjenigen der europäischen Sardine züchtete, fand ich immer 38-40 prä-anale und nicht mehr als 10 bis 12 post-anale Myotomen. Bei den Larven, welche ich aus einem demjenigen des europäischen Sprotts sehr ähnlichen Ei gezogen habe, fanden sich nur 29-30 prä-anale und ungefähr 16 post-anale Myotomen. Ausser den eben erwähnten wurde in der Javasee noch ziemlich allgemein ein Ei angetroffen, woraus eine Larve schlüpft mit 50 prä-analen und nicht mehr als 10 oder 11 post-analen Myotomen.

Wie steht es nun mit der Wirbelzahl der häufigsten Clupeiformes aus der Javasee? Ich fand hierfür folgende Zahlen:

\begin{tabular}{|c|c|}
\hline Albula vulpes & $=74 \perp$ (Bandeng tjururut) \\
\hline Chirocentrus dorab & $\perp 44+29=73 \perp$ (Parang-parang). \\
\hline Megalops cyprinoides & $\perp 38+30=68 \perp$ (Bulan-bulan). \\
\hline Elops hawaiensis & $\perp 46+21=67 \perp$ (Bandeng lelaki). \\
\hline Dussumieria hass & $\perp 41+18=59 \perp(\mathrm{Djapu})$. \\
\hline Clupea fimbriata & $\perp 29+16=45 \perp$ (Tembang). \\
\hline
\end{tabular}




$$
\begin{array}{ll}
\text { Clupea kanagurta } & \perp 27+16=43 \perp \text { (Mata belo). } \\
\text { " leiogaster } & \perp 29+14=43 \perp \text { (Lemuru). } \\
\text { Chanos chanos } & \perp 30+13=43 \perp \text { (Bandeng). } \\
\text { Dorosoma chacunda } & \perp 25+16=41 \perp \text { (Selanget). } \\
\text { Clupeoides lile } & \perp 24+16=40 \perp \text { (Tembang putih). }
\end{array}
$$

Wir fanden nun bei den Larven, worüber dieser Aufsatz handelt; im Ganzen gut 70 Myotomen, - bei der ältesten abgebildeten Larve (Fig. 6) $54+19=73$-, und es braucht demnach wohl nicht näher betont zu werden, dass bloss die ersten vier Arten der oben gegebenen Liste in Betracht kommen, wenn wir jetzt versuchen wollen, diese Larven näher zu bestimmen.

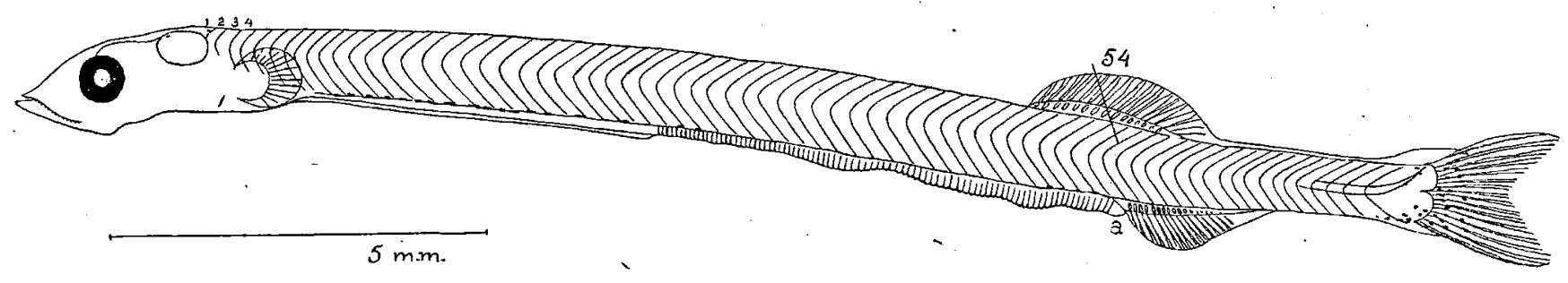

Fig. 6. Larve von 20,5 m.M.

Nun wurde für Albula vulpes, welche übrigens ziemlich selten in der Javasee gefangen wird, eine bandförmige, Leptocephalus-ähnliche Larve von GILBERT abgebildet (verg. BoulEnGER, The Cambridge Natural History, Fishes, 1904, p. 548). Diese Larve stimmt nicht mit der unseren überein, und Albula vulpes ist also auszuschliessen. Dasselbe gilt für Megalops cyprinoides, für welche VAN KAMPEN $^{1}$ ) eine ähnliche Larve beschreibt und abbildet. Wir haben also nur noch die Wahl zwischen Chirocentrus dorab und Elops hawaiensis, von welchen beiden erstere dem Genus Clupea am nächsten steht.

Die Entscheidung zwischen beiden bringt die Zahl der Flossenstrahlen der Analflosse. Verfolgen wir dazu zuerst die weitere Entwicklung. Fig. 4 zeigt eine pelagisch gefischte Larve, wobei die Augen schwarz geworden sind, was bei der Entwicklung pelagischer Fischlarven fast immer mit dem Verschwinden des Dottersackes zusammen zu fallen scheint. Auch am Körper ist schwarzes Pigment aufgetreten, besonders am Unterrande der Myotomen findet sich links und rechts vom Darm eine ganze Reihe Fleckchen, wie man sie auch bei Clupea-Arten antrifft. Dorsal finden sich ebenfalls einige solcher Fleckchen, besonders am Vorderende. Diese Fleckchen liegen jedoch in einfacher Reihe, median. Der Unterkiefer hat sich gebildet. Der Kiemendeckel wächst nach hinten aus.

Im Stadium der Fig. 5 fängt die Anlage der Dorsal- und der Caudalflosse an deutlich zu werden. Dasselbe gilt für die ringförmigen Einschnürungen des Darms, welche in Fig. 6 gut entwickelt sind. Die Ringelung des Darms gehört zu den typischen Merkmalen der Hering- und Sardellen-artigen Fischlarven. An den Kiefern treten schwache Zähnchen auf (der erwachsene Chirocentrus hat gut entwickelte, spitze Zähne).

In Fig. 6 haben Dorsal- und Caudalflosse sich weiter entwickelt und auch die Analflosse wird angelegt. In der Caudalflosse lassen sich 19 Strahlen zählen, eine Zahl welche sich sowohl bei Chirocentrus als bei Elops findet. In der Dorsalflosse zählte ich 15 Strahlen. Für Chirocentrus beträgt diese Zahl 13 grosse +3 kleine davor, für Elops 24. In der Analflosse liessen sich bis 25 Strahlen zählen, wohinten jedoch offenbar die Anlage noch mehrerer vorbereiteit wurde. Bei Chirocentrus beläuft diese Zahl 26 bis 36, bei Elops 15-16. Die Entscheidung zwischen diesen beiden kann also nicht länger zweifelhaft sein: unsere Larve gehört zu Chirocentrus dorab. Dieser Schluss wurde noch dadurch bestätigt, dass ich nachher an Eiern aus dem Ovarium eines erwachsenen Chirocentrus dieselbe feine wabenförmige Zeichnung beobachten konnte, welche für die pelagisch gefischten Eier so charakteristisch ist.

1) Kurze Notizen über Fische des Java-Meeres. 3. Die Larve von Megalops cyprinoides Brouss. in: Bulletin du Département de l'Agriculture aux Indes Neerlandaises, No 20. 
Grössere Larven als von 22 m.M. sind mir noch nicht zu Gesicht gekommen. Wir müssen annehmen, dass bei der weiteren Entwicklung die vorwärtsche Wanderung des Anus, welche sich bei den älteren bis jetzt studierten Larven konstatieren liess, Fortgang hat, sodass der Anus, welchen wir schon vom 57. oder 58. nach dem 52. Myotom vorrücken sahen, beim erwachsenen Tier schliesslich unter dem 43. oder 44. Wirbel zu liegen kommt. Ich fand nämlich für die Wirbelzahl bei 4 Exemplaren:

$$
\begin{aligned}
& 44+29=73 \\
& 43+30=73 . \\
& 44+30=74 . \\
& 43+29=72 .
\end{aligned}
$$

Resumierend können wir sagen, dass die larvale Entwicklung von Chirocentrus dorab eine grosse Uebereinstimmung mit derjenigen der Clupeiden aufweist. Die Eier stimmen mit denjenigen der Clupeiden darin überein, dass sie einen segmentierten Dotter haben. Sie sind leicht kennbar an der Anwesenheit einer Anzahl kleiner, farbloser Oeltröpfen und besonders an der feinen, netzförmigen Zeichnung der Eihaut.

Batavia, Januar 1922. 